Cahiers $d u$ MONDE RUSSE

\section{Cahiers du monde russe}

Russie - Empire russe - Union soviétique et États indépendants

$55 / 3-4 \mid 2014$

Varia

\title{
Jörn Happel, Christophe von Werdt, Mira Jovanović éds., Osteuropa kartiert - Mapping Eastern Europe
}

\author{
Sabine V. Löwis
}

\section{OpenEdition}

Journals

Édition électronique

URL : http://journals.openedition.org/monderusse/8112

DOI : $10.4000 /$ monderusse. 8112

ISSN : $1777-5388$

Éditeur

Éditions de l'EHESS

\section{Édition imprimée}

Date de publication : 1 juillet 2014

Pagination : 479-482

ISBN : 978-2-7132-2441-6

ISSN : 1252-6576

\section{Référence électronique}

Sabine V. Löwis, « Jörn Happel, Christophe von Werdt, Mira Jovanović éds., Osteuropa kartiert Mapping Eastern Europe », Cahiers du monde russe [En ligne], 55/3-4 | 2014, mis en ligne le 14 avril 2015, Consulté le 25 septembre 2020. URL : http://journals.openedition.org/monderusse/8112 ; DOI : https://doi.org/10.4000/monderusse. 8112

Ce document a été généré automatiquement le 25 septembre 2020.

2011 


\title{
Jörn Happel, Christophe von Werdt, Mira Jovanović éds., Osteuropa kartiert - Mapping Eastern Europe
}

\author{
Sabine V. Löwis
}

\section{RÉFÉRENCE}

Jörn HAPPEL, Christophe VON WERDT, Mira JOVANOVIĆ éds., Osteuropa kartiert -

Mapping Eastern Europe, Münster : LIT Verlag (Osteuropa Band 3), 2010, 364 p.

1 Ce recueil d'articles aborde des questions qui s'inscrivent dans l'actualité des débats scientifiques animant la recherche en géographie, cartographie et histoire : histoire de l'espace, représentation d'images spatiales du passé, émergence de la cartographie et de ses moyens de représentation de l'Europe de l'Est. Dans ces perspectives, l'espace est-européen apparaît comme un objet d'investigation particulièrement intéressant. En effet, bien qu'étant une construction façonnée dans l'histoire dont il n'est pas possible de déterminer définitivement les limites, l'Europe orientale nourrit une multitude d'images et de représentations, tout à la fois croisées et en concurrence.

2 L'ouvrage collectif présenté ici, édité en allemand, a pour objet une thématique, le spatial turn et un nouvel engouement pour l'espace, et une méthodologie, la déconstruction de la carte, qui n'ont encore été que rarement travaillées dans la géographie et la cartographie critique en Allemagne. Le spatial turn qui s'est opéré dans de nombreuses disciplines des sciences sociales demeure controversé dans la géographie allemande. Durant les deux dernières décennies, celle-ci a cherché à se libérer du déterminisme et de l'essentialisme spatial qui la caractérisaient, pour se tourner vers une géographie des pratiques qui attribue un nouveau rôle à l'espace matériel. Il est ainsi regrettable que l'ouvrage et ses différentes contributions ne soient pas clairement resitués dans le contexte des discussions de la cartographie critique et 
du spatial turn en histoire, discipline qui fut la première à réfléchir sur l'usage de cartes historiques.

3 L'ouvrage rassemble des articles contribuant à une cartographie et à une géographie historiques critiques de l'Europe orientale. Il s'intéresse aux formes graphiques de représentation, à leur émergence et à l'appréciation des cartes, c'est-à-dire aux questions de savoir comment et dans quels buts les contenus étaient représentés, selon quels objectifs politiques les cartes étaient produites. Cette question est particulièrement pertinente dans deux contributions sur la représentation cartographique de la Hongrie après la Première Guerre mondiale. Il apparaît ainsi que l'on ne peut pas séparer les modalités de la production de la carte et les intentions de sa publication.

4 L'article de Frithjof Benjamin Schenk porte ainsi sur les discours politiques formulés dans le cadre du développement du chemin de fer en Russie. Il montre que ce sont des craintes (comme la vulnérabilité militaire) et des projets (comme la mise en réseau des régions économiques) différents qui ont été mobilisés dans les débats depuis le début des années 1820. Les représentations politiques concernant la structure et la forme de l'espace déterminaient, par l'intermédiaire des cartes, les formes du développement du chemin de fer russe. Cette contribution ne fait pourtant pas appel aux différentes approches présentées pour expliquer les raisons dominantes ni les conditions de la construction du chemin de fer et n'expose pas les arguments politiques (ou budgétaires) qui se sont in fine imposés.

5 Jörn Happel analyse et interprète une carte allemande de 1916, probablement produite pour l'usage militaire et indiquant le tracé du chemin de fer transsibérien. De manière très pertinente, il étudie la façon dont la représentation cartographique était liée aux plans de sabotage allemands pendant la Première Guerre mondiale et offre ainsi une contextualisation particulièrement intéressante d'une carte à première vue innocente. Anton Kotenko prend pour objet l'espace national de la future Ukraine tel qu'il fut imaginé et esquissé par des intellectuels russes et ukrainophiles pendant les années 1860-1870. Il montre ainsi que les différentes constructions, plus ou moins bien fondées, d'un "espace ukrainien" ont contribué à la formation d'une "conscience ukrainienne». Il est particulièrement éclairant de voir comment les différentes représentations des différents auteurs furent négociées.

6 Le chapitre suivant analyse, à travers l'exemple de cartes historiques, les discours tenus par les contemporains sur l'étendue, les frontières et le caractère de l'Empire russe. À travers la représentation des débats et les transformations de la perception, la carte se révèle, non pas seulement une illustration acontextuelle, mais plutôt un objet prenant part à des processus historiques. Ceci est particulièrement vrai, comme le montre Lutz Häfner, pour l'établissement de l'Oural comme délimitation entre l'Europe et l'Asie. Christophe von Werdt, par contre, en discutant les premières représentations cartographiques de l'Empire russe, montre les difficultés à contextualiser une carte lorsque les sources sont insuffisantes. Toutefois, la notion de Rossica Europeana, dont il déduit l'existence par son interprétation des cartes, offre un bon point de départ pour une analyse ultérieure. D'une façon similaire, Annina Cavelti interprète des cartes de la Russie produites au XVI siècle et les symboles graphiques choisis, afin de décrypter la méthode utilisée pour leur dessin et les raisons de leur création. Kristina Küntzel-Witt analyse la présentation des résultats d'expéditions dans l'Atlantique Nord et la Sibérie au XVIII ${ }^{\mathrm{e}}$ siècle dans le contexte de la discussion très critique de ces résultats et de leur 
influence sur l'image du monde contemporain. Tous ces articles s'efforcent, avec succès, de resituer les cartes dans différentes stratégies de représentation, dans différents contextes politiques et sociaux contemporains. Ils permettent d'extraire des cartes les contextes discursifs et politiques dans lesquelles elles ont été produites, et confèrent ainsi à ces cartes un statut de source valable.

7 Le chapitre «Cartes, Frontières, Ethnies» est consacré aux possibilités méthodologiques et graphiques de produire des énoncés via les cartes. Peter Jordan discute systématiquement les moyens graphiques de la représentation de la conscience nationale ou ethnique, tout en montrant comment ces moyens servent à nier des faits avérés ou bien à les (dé)valoriser. L'article se lit ainsi comme une introduction à une lecture critique des cartes et des techniques de leur production. Les autres contributions forment, dans ce cadre, des études de cas, surtout en ce qui concerne l'exemple de la Hongrie au xxe siècle: Franz Sz. Horváth prend l'Atlas de l'Europe centrale d'András Ronai comme exemple de la façon dont la science cartographique est utilisée par une politique révisionniste hongroise dans l'entre-deux-guerres. Róbert Keményfi montre, à propos des représentations cartographiques des Carpathes comme région de l'État hongrois au début du $\mathrm{Xx}^{\mathrm{e}}$ siècle, comment l'argument des conditions naturelles était associé aux revendications économiques, sociogéographiques, politiques et territoriales. En utilisant une approche similaire, Tomasz Kamusella s'intéresse à la représentation cartographique de phénomènes ethnolinguistiques dans les atlas historiques scolaires de différents pays.

8 Le chapitre « Politique de la carte » s'ouvre avec une introduction de Jörg Stadelbauer sur le rôle des cartes comme mise en scène de l'histoire après 1945 et présente, dans trois contributions consécutives, la représentation cartographique de "l'Est allemand " de 1905 à 2008. Tobias Wegner analyse la cartographie allemande de la Pologne au xxe siècle, Antje Kempe l'usage des pictogrammes dans la représentation des territoires acquis par la Pologne, enfin Christian Lotz s'intéresse à la représentation de la frontière germano-polonaise lors des expositions internationales consacrées au tourisme. Les trois articles se caractérisent par leur intérêt pour la dimension politique de la cartographie, tout en prenant en considération les moyens graphiques accessibles aux concepteurs. Ainsi la cartographie et ses techniques apparaissent comme un savoir permettant la domination et, partant, un instrument géopolitique. Wegner démontre de manière très convaincante comment une représentation spécifique souvent répétée est efficace, encore aujourd'hui. C'est le cas, par exemple, de celles diffusées par les associations représentant les réfugiés allemands et autrichiens. Cet article engagé revendique une recherche critique et encourage l'étude des représentations et des utilisations des cartes de "l'Est allemand", avec une perspective cartographique et historique, afin de ne pas minimiser les mythes politiques du passé.

9 Kempe traite de l'enracinement symbolique des territoires intégrés dans la Pologne après la conférence de Potsdam et de la représentation par des pictogrammes des déplacements forcés de la population allemande qui suivirent. Pendant que Kempe montre comme la frontière, suivant des revendications polonaises, serait représentée de façon explicite, Lotz souligne que la cartographie touristique de l'Allemagne de l'Ouest niait ou au moins dévalorisait cette même frontière jusqu'au Traité de Varsovie de 1972, en ne la représentant pas comme une frontière entre États. Les trois contributions donnent ainsi à voir comment la cartographie était utilisée comme 
instrument politique, et comment par l'intermédiaire de cartes les positions stratégiques et politiques étaient négociées.

En conclusion de l'ouvrage, on trouvera dans le chapitre "Poésie de la carte ", une reconstruction de Cracovie par Daniel Henseler, sur la base des poèmes d'Adam Zagajewski. La description lyrique de lieux et de rues y crée une «carte mentale » très individuelle qui, en même temps, représente passé et présent, offrant ainsi un bel épilogue à l'ensemble des contributions.

11 L'ouvrage, en documentant la cartographie critique d'aujourd'hui, va bien plus loin que le spatial turn en histoire, qui se contentait jusque-là de redécouvrir la carte comme une simple source, dont la fabrication était historiquement décrite et analysée. Le risque était ainsi de négliger le fait que, si on peut « lire le temps dans l'espace », l'espace ne peut pas être assimilé à ce que montrent les cartes. La cartographie présente une construction de représentations spatiales et non un espace réel dans lequel se déroulerait l'histoire. L'ouvrage montre très clairement, à l'exemple de l'Europe de l'Est, comment s'effectue cette production de la représentation des constructions spatiales dans la cartographie. Ces représentations servent en partie à une structuration réelle de l'espace, mais aussi à médiatiser, à imposer et à populariser des conceptions (politiques, infrastructurelles, mentales) de l'espace. Dans ce sens, cet ouvrage intéressant remplit pleinement le but qu'il s'est donné d'étudier la méthodologie et la valeur des cartes comme source historique.

\section{AUTEURS}

\section{SABINE V. LÖWIS}

Centre Marc Bloch, Humboldt-Universität, Berlin 